\title{
Morphology-Dependent Energy Transfer of Polyfluorene Nanoparticles Decorating InGaN/GaN Quantum-Well Nanopillars
}

\author{
Talha Erdem, ${ }^{\dagger}$ Vusala Ibrahimova, ${ }^{\dagger}$ Dae-Woo Jeon, ${ }^{\ddagger}$ In-Hwan Lee, ${ }^{\ddagger}$ Dönüs Tuncel, ${ }^{\dagger}$ \\ and Hilmi Volkan Demir ${ }^{*} \dagger, \S$
}

\begin{abstract}
${ }^{\dagger}$ Departments of Electrical and Electronics Engineering, Physics, and Chemistry, UNAM-National Nanotechnology Research Center, and Institute of Materials Science and Nanotechnology, Bilkent University, Ankara 06800, Turkey

${ }^{\ddagger}$ School of Advanced Materials Engineering, Research Center of Industrial Technology, Chonbuk National University, Chonju 561-756, Republic of Korea

${ }^{\S}$ School of Electrical and Electronic Engineering and School of Physical and Mathematical Sciences, Nanyang Technological University, Singapore 639798, Singapore
\end{abstract}

\section{Supporting Information}

\begin{abstract}
Conjugated polymer nanoparticles (CPNs), prepared in aqueous dispersion from poly[(9,9-bis $\{3-$ bromopropyl $\}$ fluorenyl-2,7-diyl)-co-(1,4-benzo- $\{2,1,3\}$-thiodiazole)] (PFBT-Br), are incorporated into a nanopillar architecture of InGaN/GaN multiple quantum wells (MQWs) to demonstrate a new organic/inorganic class of nanostructured excitonic model system. This hybrid system enables intimate integration for strong exciton-exciton interactions through nonradiative energy transfer (NRET) between the integrated CPNs and MQW pillars. The NRET of these excitonic systems is systematically investigated at varied

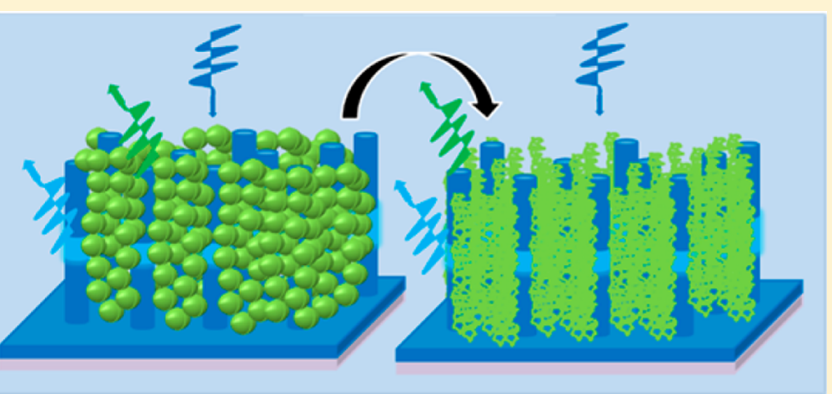
temperatures. In these hybrids, InGaN/GaN MQWs serve as the donor of the NRET pair, while immobilized PFBT-Br polymer serves as the acceptor. To understand morphology-dependent NRET, PFBT-Br CPNs coating InGaN/GaN MQWs are made to defold into polymer chains by in situ treatment with a good solvent (THF). The experimental results indicate that NRET is significantly stronger in the case of CPNs compared with their defolded polymer chains. At room temperature, while the NRET efficiency of open polymer chains-nanopillar system is only 10\%, PFBT-Br CPNs exhibit a substantially higher NRET efficiency of $33 \%$ (preserving the total number of polymer molecules). The NRET efficiency of the nanoparticle systems is observed to be $25 \%$ at $250 \mathrm{~K}, 22 \%$ at $200 \mathrm{~K}, 19 \%$ at $150 \mathrm{~K}$, and $15 \%$ at $100 \mathrm{~K}$. On the other hand, the defolded polymer chains exhibit significantly lower NRET efficiencies of $17 \%$ at $250 \mathrm{~K}, 16 \%$ at $200 \mathrm{~K}, 11 \%$ at $150 \mathrm{~K}$, and $5 \%$ at $100 \mathrm{~K}$. This work may potentially open up new opportunities for the hybrid organic/inorganic systems where strong excitonic interactions are desired for light generation, light harvesting, and sensing applications.
\end{abstract}

$\mathrm{C}$ onjugated polymer nanoparticles (CPNs) attract significant attention for important applications including bioimaging, ${ }^{1-3}$ biosensing, ${ }^{4,5}$ and optoelectronics. ${ }^{6-12}$ One of the most attractive features of CPNs is the convenient tunability and control of their properties through the choice and functionalization of the polymer and the surface modification of nanoparticles. Furthermore, CPNs exhibit low toxicity, ${ }^{13}$ and their mechanical stability can be enhanced through cross-linking. ${ }^{14}$ Their optical properties arise from the controlled conformational changes of the polymer and their aggregation form rather than the quantum confinement effects, in contrast with inorganic nanoparticles, for example, colloidal semiconductor quantum dots (QDs). ${ }^{13}$ As a result of these attractive properties, CPNs find use as alternative color convertors, or molecular beacons, in various biotechnology ${ }^{1,3,4}$ and optoelectronic applications. ${ }^{6,9,10,13,14}$

The emission of color convertors, including CPNs, can be enhanced via the Förster-type nonradiative energy transfer
(NRET) (also dubbed as Förster resonance energy transfer, FRET), which basically relies on the exciton-exciton interactions between the donor and acceptor emitters. ${ }^{15}$ For example, polymers, ${ }^{16}$ organic dyes, ${ }^{17}$ inorganic QDs, ${ }^{18}$ and epitaxial quantum wells ${ }^{18,19}$ have been widely investigated for NRET studies. CPNs were also used in similar studies because of their preferably high absorption cross sections. ${ }^{20,21}$

Another important class of materials in optoelectronics is the III-nitrides. They are the main building blocks of today's UV, blue, green, and white light-emitting diodes. Using these materials, a wide range of spectral regions from the ultraviolet to the green can be covered by controlling their alloy composition. In particular, quantum-well (QW) architectures

Received: May 2, 2013

Revised: August 14, 2013

Published: August 14, 2013 
are of great importance because they enable spectral tuning and enhancement in the emission quantum efficiency. Because InGaN/GaN QWs having a wide bandgap are used for highenergy photon generation, they are also valuable for NRET studies. In the literature this type of QWs was used together with both organic and inorganic luminophors. For example, epitaxial QWs were hybridized with polymers, ${ }^{22}$ dyes, $^{23}$ and other organic molecules. ${ }^{24}$ As an example of inorganic material integration on QWs, Achermann et al. used epitaxial QWs with semiconductor nanocrystal QDs and reached a transfer efficiency of $65 \% .^{18}$ Later, Chanyawadee et al. achieved an NRET efficiency of $82 \%$ using nanocrystal QDs and QWs having elliptical holes reaching down their wells. ${ }^{25}$ In an independent work of Nizamoglu et al., the use of nanopillars housing QWs was also reported to increase the NRET efficiency to $83 \%{ }^{26}$ The enhancement in the NRET efficiency is basically enabled by the reduced distance between the QWs and the colloidal QDs in these examples.

In this work, different from the previous reports of our group and others, we studied aqueous dispersions of CPNs made of poly[(9,9-bis \{3-bromopropyl\}fluorenyl-2,7-diyl)-co-(1,4-benzo$\{2,1,3\}$-thiodiazole)] (PFBT-Br), whose chemical structure is given in Figure 1, immobilized into InGaN/GaN QW

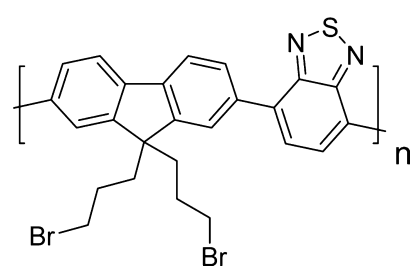

Figure 1. Molecular structure of poly[(9,9-bis\{3-bromopropyl $\}$ fluorenyl-2,7-diyl)-co-(1,4-benzo-\{2,1,3\}-thiodiazole)] (PFBT-Br).

nanopillar architecture to make a new class of hybrid excitonic model systems consisting of organic and inorganic nanostructures. We observed that nanopillars transfer their excitation energies to CPNs with a substantially higher efficiency compared with their defolded polymer chains at varying temperatures (at 296, 250, 200, 150, and $100 \mathrm{~K}$ ), while preserving the same number of polymer molecules in the system. We also observed that the NRET efficiency and rate are functions of temperature and decrease with decreasing temperature as a result of stronger exciton localization within InGaN/GaN QW nanopillars at lower temperatures in the case of CPN hybridization. Also, to further identify the effects of morphology directly on the emission kinetics of organic molecules used in this study, we examined the emission decay characteristics of PFBT-Br CPNs and their defolded polymer chains on a quartz substrate. We found that the emission kinetics of CPNs decorating nanopillars and those on quartz are similar, whereas the defolded polymer chains exhibit completely different behavior. This study shows that the emission dynamics of CPNs are more immune to environmental and morphological changes than defolded polymer chains are.

\section{MATERIALS AND EXPERIMENTAL METHODOLOGY}

Synthesis of PFBT-Br. 2,1,3-Benzothiadiazole-4,7-bis(boronic acid pinocol ester) (411 mg, $1.06 \mathrm{mmol}$ ), 2,7dibromo-9,9-bis(3-bromo-propyl)-9H-fluorene (566 mg, 1.06 $\mathrm{mmol})$, and $\mathrm{K}_{2} \mathrm{CO}_{3}(1.47 \mathrm{~g}, 10.6 \mathrm{mmol})$ were dried under vacuum for $\sim 30 \mathrm{~min}$. Subsequently, degassed solvents, THF $(10 \mathrm{~mL})$, water $(10 \mathrm{~mL})$, and toluene $(10 \mathrm{~mL})$ were added to the mixture under argon gas. Then, the catalyst, tetrakis(triphenylphosphine) palladium $\left[\mathrm{Pd}\left(\mathrm{PPh}_{3}\right)_{4}\right]$, was quickly added. After $3 \mathrm{~h}$ of stirring of the mixture under argon at 80-90 ${ }^{\circ} \mathrm{C}$, the phase-transfer catalyst, tetra- $n$-butylammonium bromide (TBAB), was added. The stirring was continued for another $48 \mathrm{~h}$ at $80-90{ }^{\circ} \mathrm{C}$ to complete the polymerization reaction. The mixture was evaporated under vacuum to obtain a solid residue, which was suspended in water; the waterinsoluble particles were then collected by suction and dissolved in THF $(15 \mathrm{~mL})$, and the solution was precipitated into cold methanol $(200 \mathrm{~mL})$. The precipitates were collected by suction and dried under vacuum for $6 \mathrm{~h}(547 \mathrm{mg}, 56 \%)$. The synthesis of PFBT-Br can also be found in ref 27. ${ }^{1} \mathrm{H}$ NMR (400 MHz, $\left.\mathrm{CDCl}_{3}, \delta\right) 7.99(\mathrm{~m}, 8 \mathrm{H}, \mathrm{Ar}-\mathrm{H}), 3.35$ (q, 4H, $\left.\mathrm{CH}_{2} \mathrm{Br}\right), 2.41$ (q, $\left.4 \mathrm{H}, \mathrm{CH}_{2}\right), 1.25\left(\mathrm{~m}, 4 \mathrm{H}, \mathrm{CH}_{2}\right)$. GPC: $M_{\mathrm{n}}=4.5 \times 10^{3} \mathrm{~g} / \mathrm{mol}$, $M_{\mathrm{w}}=1.1 \times 104 \mathrm{~g} / \mathrm{mol}$ (polystyrene as standard).

Preparation of PFBT-Br Nanoparticles. Nanoparticles were prepared by the reprecipitation method. ${ }^{13} 2 \mathrm{mg}$ of PFBT$\mathrm{Br}$ polymer was dissolved in $10 \mathrm{~mL}$ of THF and ultrasonicated for $15 \mathrm{~min}$. After sonication, the solution was filtrated via syringe filter and injected into rapidly stirring $100 \mathrm{~mL}$ of Milli$\mathrm{Q}$ water $(18.2 \mathrm{M} \Omega)$ and ultrasonicated for another $1 \mathrm{~h}$. Vacuum rotary evaporator was used to remove THF to obtain stable nanoparticles. Dynamic light scattering (DLS) measurements showed that the average diameter of the synthesized CPNs is $69 \mathrm{~nm}$. Absorption and fluorescence spectra of the polymer and nanoparticles are given in Figure 2a, and DLS results are given in Table S1 and Figure S1a in the Supporting
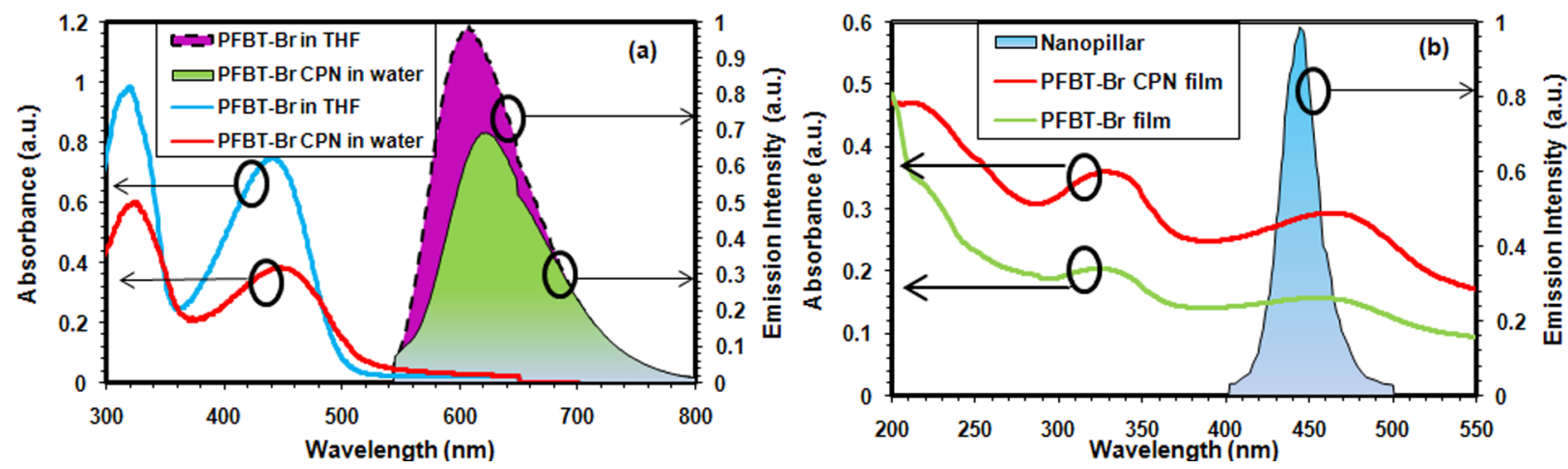

Figure 2. (a) Absorbance and fluorescence spectra of PFBT-Br in THF and PFBT-BR CPNs in water and (b) absorbance spectra of PFBT-Br CPN film and defolded PFBT-Br (while preserving the amount of polymers on quartz), together with the photoluminescence of InGaN/GaN QWs. 
Information (SI). Absorption spectrum of PFBT-Br film on quartz is also recorded (Figure S1b in the SI). We observed that there is no major difference in the photoluminescence between PFBT-Br films prepared directly and prepared by adding THF onto PFBT-Br CPN film and drying it.

Epitaxial Growth of InGaN/GaN Quantum Wells. InGaN/GaN multiple QWs were grown on a sapphire substrate using metal organic chemical vapor deposition (MOCVD). The precursors of $\mathrm{Ga}$, In, and $\mathrm{N}$ were trimethylgallium (TMGa), trimethylindium (TMIn), and $\mathrm{NH}_{3}$, respectively. First the cplane sapphire substrate was annealed at $1000{ }^{\circ} \mathrm{C}$ for $10 \mathrm{~min}$. Subsequently, a buffer layer of $\mathrm{GaN}$ was grown at low temperature. Following the buffer layer, an undoped $\mathrm{GaN}$ layer $(1 \mu \mathrm{m})$ and $\mathrm{n}$-type $\mathrm{GaN}$ layer $(2 \mu \mathrm{m})$ were grown at 1060 ${ }^{\circ} \mathrm{C}$. Five pairs of InGaN/GaN QWs were then grown on top of this n-type layer. Finally, a $150 \mathrm{~nm}$ thick GaN layer was grown to complete the epitaxial structure.

Preparation of InGaN/GaN QW Nanopillars. A $100 \mathrm{~nm}$ thick $\mathrm{SiO}_{2}$ was deposited by plasma-enhanced chemical vapor deposition (PECVD) on the epitaxially grown layers, followed by a $10 \mathrm{~nm}$ thick $\mathrm{Ni}$ thin-film deposition using an e-beam evaporator. The formation of $\mathrm{Ni}$ clusters was realized upon annealing the epi-wafer at $800{ }^{\circ} \mathrm{C}$ for 1 min under $\mathrm{N}_{2}$ flow. $\mathrm{SiO}_{2}$ and $\mathrm{GaN}$ layers were etched using inductively coupled plasma-reactive ion etching (ICP-RIE). $\mathrm{Ni}$ and $\mathrm{SiO}_{2}$ regions were then removed using buffered oxide etchant (BOE). Surface passivation was carried out via $\mathrm{KOH}$ and $\mathrm{NH}_{4} \mathrm{~S}$ treatment and confirmed by an increased intensity compared with both nontreated and planar cases. The photoluminescence spectrum of nanopillars is given in Figure $2 b$.

Experimental Procedure. First, time-resolved and steadystate photoluminescence measurements of the InGaN/GaN nanopillars were taken at 100, 150, 200, 250, and $296 \mathrm{~K}$. Then, PFBT-Br CPNs having an average diameter of $69 \mathrm{~nm}$ were integrated with the nanopillars via careful drop-casting and dried at $50{ }^{\circ} \mathrm{C}$. Time-resolved and steady-state fluorescence measurements were taken at the same temperatures. Subsequently, the CPNs decorating the nanopillars were converted into the polymer chains by adding small amounts of THF and then fully evaporating it. By doing so, we aimed to preserve the number of acceptor molecules in the hybrid system and make a fair comparison between both of the cases using exactly the same polymer chains. This experimental methodology is illustrated in Figure 3. To ensure the validity of our results, it

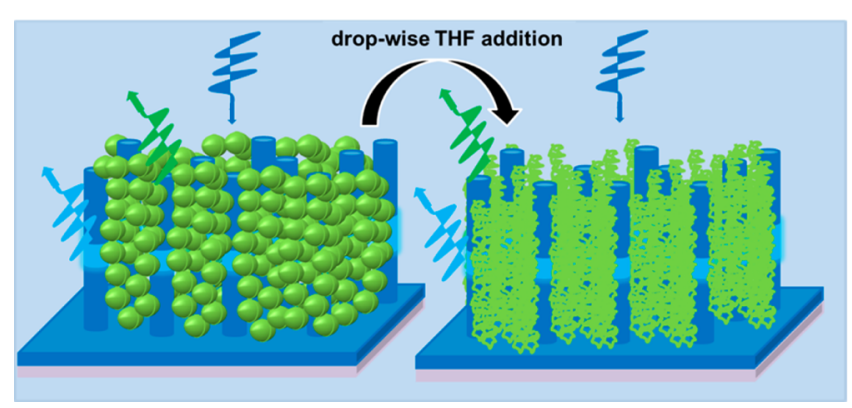

Figure 3. Illustration of the comparative study: First aqueous dispersion of nanoparticles is drop-casted on the nanopillars and cryogenic time-resolved and steady state fluorescence measurements are taken at $296,250,200,150$, and $100 \mathrm{~K}$. Later, THF is dropwise added onto the nanoparticles on the nanopillars to defold them into polymer chains and then fully evaporated. The same measurements are repeated for the case of open polymers. is necessary to make sure that there are enough acceptors surrounding the QWs. For this purpose, we carefully prepared the samples and took their SEM image, including CPNs immobilized around nanopillars (Figure S2a in the SI) as well as the THF-treated version of the hybrid structure in which the CPNs were made to defold into polymer chains using and fully evaporating THF (Figure S2b in the SI). As can be seen from the images of both cases, nanopillars are just fully covered. In addition, the same amount of PFBT-Br CPNs was also dropcasted on a quartz substrate, and later THF was added onto this film while again preserving the same number of polymer molecules. Consequently, this systematic study allowed us to analyze the emission and NRET kinetics of these two different architectures of PFBT-Br molecules (as CPNs and polymer chains). Time-resolved fluorescence decays of all of these films were also recorded at the corresponding temperatures. The lifetimes were determined by fitting multiexponential functions to the experimentally measured decay curves. In all of the cases, $\chi^{2}$ remained within 0.9 and 1.1 , mostly very close to 1.0 , which indicates a strong correlation between the experimental data and the fitted exponential functions. The error in the lifetimes was calculated to be $<7.6$ and $<5 \%$ for the nanopillars and acceptor molecules, respectively.

\section{RESULTS AND DISCUSSION}

Aqueous dispersions of CPNs were prepared from PFBT-Br ${ }^{27}$ using the reprecipitation method in which the polymer chains are collapsed into spherical nanoparticles upon exposure to a poor solvent (e.g., water). The average size of the resultant CPNs was determined to be $69 \mathrm{~nm}$ using DLS measurement (Figure S1a and Table S1 in the SI). The fluorescence and absorbance spectra of PFBT-Br in THF and PFBT-Br CPNs in water are given in Figure 2a. PFBT-Br CPNs were incorporated into the nanopillars to obtain nanostructured hybrid systems in which $\mathrm{InGaN} / \mathrm{GaN}$ QWs serve as the donor and CPNs serve as the acceptor in the NRET. The donor peak emission wavelength is $\sim 450 \mathrm{~nm}$, whereas the peak emission wavelength of the acceptor is located around $550 \mathrm{~nm}$. Following the characterization of the InGaN/GaN-CPN hybrids, CPNs coating the multiple quantum well (MQW) nanopillars were made to defold in situ into polymer chains by carefully treating with a good solvent (e.g., THF), which allows for preserving the amount of polymers to study the morphology-dependent energy transfer and emission kinetics of the hybrid system. The absorbance spectra of the PFBT-Br CPN and defolded PFBT$\mathrm{Br}$ are presented in Figure $2 \mathrm{~b}$ along with the photoluminescence spectrum of the InGaN/GaN nanopillars. The experimental procedure is illustrated in Figure 3, and the scanning electron microscopy (SEM) images of the hybrid system before and after THF treatment are given in Figure S2 in the SI.

$\operatorname{NRET}$ efficiency $(\eta)$ and NRET rate $\left(\gamma_{\mathrm{NRET}}\right)$ are calculated using eqs 1 and 2, respectively. Here $\tau_{\mathrm{DA}}$ stands for the lifetime of donors in the presence of acceptors and $\tau_{\mathrm{D}}$ denotes the lifetime of donors in the absence of acceptors.

$$
\begin{aligned}
& \eta=1-\frac{\tau_{\mathrm{DA}}}{\tau_{\mathrm{D}}} \\
& \gamma_{\mathrm{NRET}}=\frac{1}{\tau_{\mathrm{DA}}}-\frac{1}{\tau_{\mathrm{D}}}
\end{aligned}
$$

The corresponding lifetimes of the nanopillar-PFBT-Br hybrid systems at the donor peak emission wavelength are 

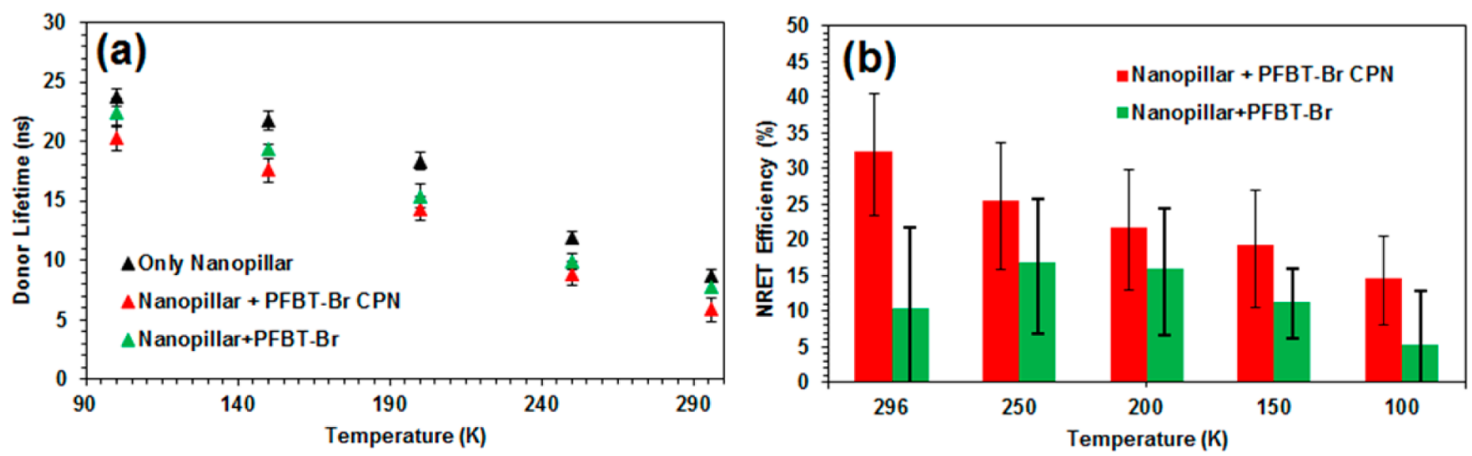

Figure 4. Temperature-dependent lifetimes of (a) nanopillars alone, nanopillars in the presence of PFBT-Br CPNs, and nanopillars in the presence of defolded PFBT-Br polymer chains and (b) temperature-dependent NRET efficiency of the nanopillar-CPN hybrid and nanopillar-defolded PFBTBr hybrid. Black error bars indicate the measurement errors for (a), and the error bars in (b) were calculated using the information in panel a. The minimum NRET efficiency was calculated by taking the minimum lifetime value of nanopillar alone and the maximum donor lifetime of acceptor including system. Similarly, the maximum NRET efficiency was found by using the maximum lifetime value of nanopillar alone and the minimum donor lifetime of acceptor including system.
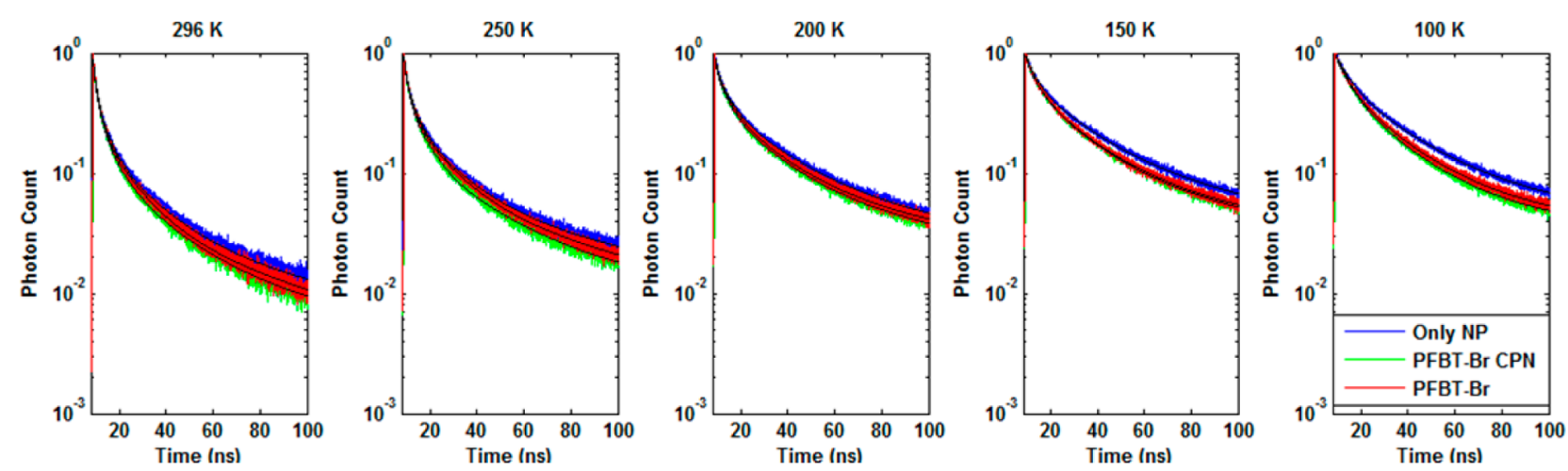

Figure 5. Time-resolved fluorescence decay curves of the nanopillars alone, the nanopillars hybridized with PFBT-Br CPNs and those hybridized with PFBT-Br polymer (after the addition and evaporation of THF) at $450 \mathrm{~nm}$ at temperatures of 296, 250, 200, 150, and $100 \mathrm{~K}$. Black curves within the colored decay curves are the multiexponential functions numerically fitted to the experimental data.

given in Figure 4 and listed in Table S2 in the SI, together with the energy-transfer efficiencies for CPNs and defolded polymer chains. Additionally, time-resolved fluorescence decay curves are presented at the donor and acceptor peak emission wavelengths in Figure 5 and Figure S3 in the SI, respectively.

We observe a significant difference in the donor lifetimes and, consequently, in the energy-transfer efficiencies after both (i) PFBT-Br CPN hybridization on InGaN/GaN nanopillars and (ii) PFBT-Br polymer chains obtained by defolding these CPNs via THF addition. Furthermore, the average donor lifetimes in the case of CPNs shorten more after hybridization than in the case of the defolded polymers. This further lifetime shortening of nanopillars can possibly stem from the following: (a) an increase in the distance between the QWs of donor nanopillars and acceptor molecules after defolding, (b) a more successful surface passivation by defolded polymers compared with CPNs, or (c) a more successful energy-transfer process in the case of CPNs compared with defolded polymer chains. Here the first problem was avoided during the experiment by dropwise addition of THF without removing the defolded polymers away from the nanopillars. The SEM imaging revealed that the nanopillars were fully covered by CPNs before the THF treatment and a significant surface coverage was achieved after THF addition (Figure S2 in the SI). With great care taken not to remove the polymers, the defolded polymers come even closer to the active region of the nanopillars. If the defolded polymers had the same NRET characteristics as the CPNs, then one would expect to observe a stronger decrease in the lifetime of the nanopillars compared with CPNs.

As the second possibility, the surface passivation issue was addressed during the preparation of samples. To eliminate this problem, we carefully passivated nanopillar surfaces via $\mathrm{KOH}$ and $\mathrm{NH}_{4} \mathrm{~S}$ surface treatment during the fabrication process. The proper passivation was verified by the increased intensity $(I)$ of the nanopillars with respect to the planar case. We observed that the emission intensities are ordered in magnitude as $I_{\text {beforetreatment }}<I_{\text {planar }}<I_{\text {aftertreatment, }}$ and the peak emission wavelength, which blue-shifts with the nanopillar etching, stays constant before and after the surface treatment, that is, $\lambda_{\text {planar }}>$ $\lambda_{\text {beforetreatment }} \approx \lambda_{\text {aftertreatment. This means that the nanopillar }}$ etching leads to strain relaxation, which is expected to blue-shift the peak emission and increase the emission intensity. Once the nanopillars are formed, the surface treatment does not affect the spectral content of the emission but only its intensity. This implies that the surface states were in fact effective before the surface treatment, reducing the emission intensity substantially before passivating the pillar surfaces.

As a result, here we attribute this stronger lifetime shortening of nanopillars mainly to NRET process; however, an error analysis is still necessary. Such an analysis should be carried out considering Figures $4 b$ and 5 together. According to Figure $4 b$, the minimum NRET efficiency of the nanopillar-CPN hybrid system at room temperature turns out to be higher than the 
maximum NRET efficiency of the defolded CPN hybrid system. At 250 and $200 \mathrm{~K}$, the NRET efficiency error bars overlap more strongly, which is a consequence of the increasing NRET efficiency of the defolded CPN hybrid system together with the decrease in the NRET efficiency of the CPN hybrid system. A similar conclusion can be drawn by investigating the decay curves in Figure 5, where the decay curves of both hybrid systems get closer. Nevertheless, the CPN hybrid system is observed to attain a faster decaying trend at these two temperatures and at 150 and $100 \mathrm{~K}$ as well, although the overlap of the decays gets stronger. Considering this observation, we believe that assigning a higher NRET to CPN hybrid system is still qualitatively reasonable, especially down to $200 \mathrm{~K}$, because the faster decay is a clear trend rather than noisy data points.

According to the analyses of the measured average lifetimes, the maximum NRET efficiency for the CPN hybridization is calculated to be $33 \%$ at room temperature, whereas this value drops to $10 \%$ when the nanoparticles are defolded into polymer chains via THF addition and evaporation. This is an indicator that here the CPNs within the nanopillars constitute a better NRET donor-acceptor pair compared with polymer chains. The absorption spectrum of CPNs yields 1.8 times higher overlap-integral $^{15}$ with the emission spectrum of nanopillars compared with that of defolded polymers (Figure 2b). However, our calculations reveal that this difference in the overlap integral can increase the NRET efficiency at most by 1.7 fold, which is not enough to explain the almost three-fold enhancement at room temperature. We reckon that the modified photophysical properties and exciton dynamics in the CPNs compared with the defolded polymer may play an important role in this observation. Other acceptor-related factors such as the refractive index and dipole orientation factors, which are closely related to the stacking, in other words, morphology of the acceptor, should also have an important contribution to the change of the NRET efficiency.

While decreasing temperature, we further observe that NRET rate of the CPNs is a strong function of temperature $\left(0.055 \mathrm{~ns}^{-1}\right.$ at $296 \mathrm{~K}, 0.029 \mathrm{~ns}^{-1}$ at $250 \mathrm{~K}, 0.015 \mathrm{~ns}^{-1}$ at $200 \mathrm{~K}$, $0.011 \mathrm{~ns}^{-1}$ at $150 \mathrm{~K}$, and $0.007 \mathrm{~ns}^{-1}$ at $100 \mathrm{~K}$ ) and NRET efficiencies of the system follow a slowly decreasing trend while taking the values of $33,25,22,19$, and $15 \%$ at the same temperatures, respectively (Figure 4 ). This observation can be explained by the slightly stronger localization of excitons within QWs as the temperature decreases. Further investigation of the decay dynamics reveals that the lifetime of the bare nanopillar case $\left(\tau_{\mathrm{D}}\right)$ increases as the temperature decreases to $100 \mathrm{~K}$. This is an expected behavior because of the suppression of the nonradiative transitions within the nanopillar as the temperature decreases. In the case that THF is added and defolded polymers are obtained, NRET rates decrease strongly to 0.013 $\mathrm{ns}^{-1}$ at $296 \mathrm{~K}, 0.017 \mathrm{~ns}^{-1}$ at $250 \mathrm{~K}, 0.010 \mathrm{~ns}^{-1}$ at $200 \mathrm{~K}, 0.006$ $\mathrm{ns}^{-1}$ at $150 \mathrm{~K}$, and $0.002 \mathrm{~ns}^{-1}$ at $100 \mathrm{~K}$, while the NRET efficiency first increases from 10 to $17 \%$ when the temperature decreases from room temperature to $250 \mathrm{~K}$ and remains closer to this value $(16 \%)$ at $200 \mathrm{~K}$. However, further decrease in temperature to 150 and $100 \mathrm{~K}$ decreases the NRET efficiency again down to 11 and $6 \%$, respectively. Nevertheless, the error bars of the NRET efficiencies calculated using the maxima and minima of the lifetimes reveal a strong overlap of the efficiencies. This prevents a healthy quantitative discussion on the change of morphology-dependent energy-transfer efficiency with respect to temperature. However, a qualitative analysis based on the decay curves reveals that the PFBT-Br exhibits a strong morphology-dependent energy-transfer character. On the basis of the qualitative discussion carried out above, one can conclude that the realization of a higher NRET efficiency is highly probable with the PFBT-Br CPN hybridization of inorganic In GaN/GaN nanopillars compared with their hybridization with defolded CPNs, especially at room temperature and 150 and $100 \mathrm{~K}$. Here, although the change at cryogenic temperatures cannot be quantitatively assigned to the variation in the absorption due to the inability of our measurement setup, it was possible to quantitatively relate this behavior at room temperature partially to the difference in the spectral overlap integral in addition to other factors. We consider that the observed behavior may also occur due to the temperature-related morphology changes of PFBT-Br, leading to changes in its photophysical properties (mainly the available electronic states and the absorption), and the changes in the dipole orientation factor and the refractive index might contribute as well.

In the case that CPNs were hybridized and later defolded on nanopillars, we observe that the acceptor lifetimes $\left(\tau_{\mathrm{A}}\right)$ increase monotonically and substantially as the temperature decreases. This can be an indicator for the dominance of the nonradiative recombinations in the films at room temperature because of the suppression of nonradiative transitions as the temperature decreases. For comparison purposes, the same lifetime characterization of PFBT-Br CPNs and polymer chains was repeated using quartz substrate. The corresponding lifetimes are shown in Figure 6; the tabulated values can be found in

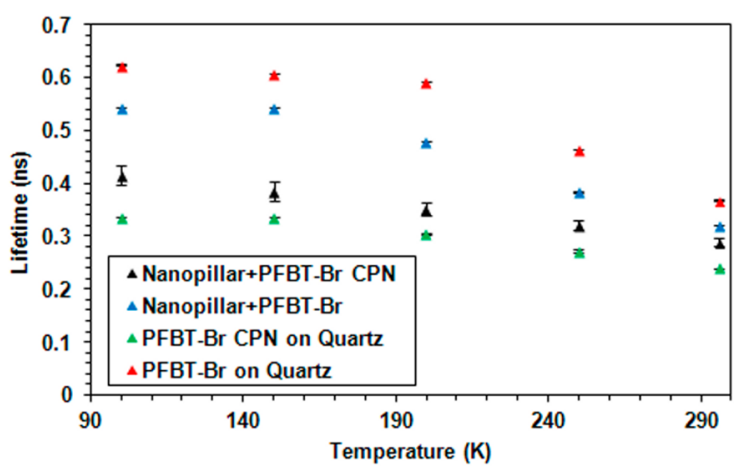

Figure 6. Temperature-dependent fluorescence lifetimes of PFBT-Br CPNs and defolded PFBT-Br on the nanopillars and quartz.

Tables S2 and S3 in the SI, and the decay curves are presented in Figure S3 in the SI. Here we observe that the lifetimes of the nanoparticles on quartz increase monotonically as the temperature decreases. This monotonic increase in the lifetime with the decreasing temperature is also consistent with the behavior of polymer chains generated by defolding the CPN structure into polymer chains. Nevertheless, the polymers exhibit much longer lifetimes compared with CPNs, showing that CPN morphology enables additional nonradiative recombination pathways, whereas these pathways are suppressed in the defolded form of polymer chains on quartz. When compared with the acceptor lifetimes on nanopillars, we observe that the CPNs exhibit similar lifetimes on quartz, whereas defolded PFBT-Br films have completely different decay lifetimes. This observation clearly shows that the emission dynamics of PFBT$\mathrm{Br}$ CPNs are less morphology-dependent than that of defolded PFBT-Br. 


\section{CONCLUSIONS}

In this work, we studied the hybrid excitonic structures of both PFBT-Br nanoparticles and their defolded polymer chains intimately integrated on the nanopillars of $\mathrm{InGaN} / \mathrm{GaN}$ multiple QWs and investigated the temperature-dependent NRET dynamics in these two hybrid architectures. We found that the NRET from the QWs to the PFBT CPNs is more efficient compared with the defolded PFBT-Br polymer chains at the temperatures tested in this study. At room temperature, the CPNs exhibit the maximum NRET efficiency of 33\%, whereas this value decreases to $10 \%$ in the case of polymer chains. Another finding of this study is that the efficiency of the energy transfer decrease as the temperature decreases for the CPNs, while the NRET efficiency shows an oscillating behavior in the case of defolded polymer chains. For comparison purposes, we further investigated the emission kinetics of the nanoparticles and polymer chains on the nanopillars and on a flat quartz substrate. We observed that in both types of substrates the nanoparticles reveal similar behavior. However, the open polymer chains are observed to feature significantly changing emission kinetics depending on the substrate. In conclusion, this work demonstrates an efficient excitonic hybrid model system made of organic and inorganic nanostructured components, enabling new possibilities for future excitonic systems in light generation and harvesting as well as sensing.

\section{ASSOCIATED CONTENT}

\section{S Supporting Information}

Additional figures (Figures S1 and S2) and tables (Tables S1, S2, and S3). This material is available free of charge via the Internet at http://pubs.acs.org.

\section{AUTHOR INFORMATION}

\section{Corresponding Author}

*E-mail: volkan@stanfordalumni.org.

\section{Author Contributions}

The manuscript was written through contributions of all authors. All authors have given approval to the final version of the manuscript.

\section{Funding}

The work was supported by ESF EURYI, EU FP7 Nanophotonics4Energy NoE, and TUBITAK under the project no. EEEAG 109E002, 109E004, 110E010, 110E217, and TBAG $210 \mathrm{~T} 139$ and in part by NRF-CRP6-2010-02 and NRF RF 2009-09.

\section{Notes}

The authors declare no competing financial interest.

\section{ACKNOWLEDGMENTS}

We acknowledge ESF EURYI, EU FP7 Nanophotonics4Energy NoE, and TUBITAK under the project no. EEEAG 109E002, 109E004, 110E010, and 110E217, and TBAG 210T139 and in part by NRF-CRP6-2010-02 and NRF RF 2009-09. H.V.D. acknowledges additional support from TUBA-GEBIP and T.E. acknowledges TUBITAK BIDEB. This material is available free of charge via the Internet at http://pubs.acs.org.

\section{ABBREVIATIONS}

NRET, nonradiative energy transfer; PFBT-Br, poly[(9,9-bis\{3bromopropyl $\}$ fluorenyl-2,7-diyl)-co-(1,4-benzo- $\{2,1,3\}$-thiodiazole)]; CPN, conjugated polymer nanoparticle; $\tau_{\mathrm{D}}$, donor lifetime in the absence of acceptors; $\tau_{\mathrm{DA}}$, donor lifetime in the presence of acceptors; $\tau_{\mathrm{A}}$ acceptor lifetime; MQW, multiple quantum well; RIE, reactive ion etching; DLS, dynamic light scattering; NMR, nuclear magnetic resonance

\section{REFERENCES}

(1) Rahim, N. A. A.; McDaniel, W.; Bardon, K.; Srinivasan, S.; Vickerman, V.; So, P. T. C.; Moon, J. H. Conjugated Polymer Nanoparticles for Two-Photon Imaging of Endothelial Cells in a Tissue Model. Adv. Mater. 2009, 21, 3492-3496.

(2) Moon, J. H.; McDaniel, W.; MacLean, P.; Hancock, L. E. LiveCell-Permeable Poly(p-phenylene ethynylene). Angew. Chem., Int. Ed. 2007, 46, 8223-8225.

(3) Howes, P.; Thorogate, R.; Green, M.; Jickells, S.; Daniel, B. Synthesis, Characterisation and Intracellular Imaging of PEG Capped BEHP-PPV Nanospheres. Chem. Commun. 2009, 2490-2492.

(4) Wu, C.; Bull, B.; Christensen, K.; McNeill, J. Ratiometric SingleNanoparticle Oxygen Sensors for Biological Imaging. Angew. Chem., Int. Ed. 2009, 48, 2741-2745.

(5) Moon, J. H.; MacLean, P.; McDaniel, W.; Hancock, L. F. Conjugated Polymer Nanoparticles for Biochemical Protein Kinase Assay. Chem. Commun. 2007, 4910-4912.

(6) Piok, T.; Gamerith, S.; Gadermaier, C.; Plank, H.; Wenzl, F. P.; Patil, S.; Montenegro, R.; Kietzke, T.; Nehrer, D.; Scherf, U.; et al. Organic Light-Emitting Devices Fabricated from Semiconducting Nanospheres. Adv. Mater. 2003, 15, 800-804.

(7) Kietzke, T.; Nehrer, D.; Landfester, K.; Montenegro, R.; Güntner, R.; Scherf, U. Novel Approaches to Polymer Blends Based on Polymer Nanoparticles. Nat. Mater. 2003, 2, 408-412.

(8) Kietzke, T.; Nehrer, D.; Kumke, M.; Montenegro, R.; Landfester, K.; Scherf, U. A Nanoparticle Approach to Control the Phase Separation in Polyfluorene Photovoltaic Devices. Macromolecules 2004, $37,4882-4890$

(9) Ozel, I. O.; Ozel, T.; Demir, H. V.; Tuncel, D. Non-radiative Resonance Energy Transfer in Bipolymer Nanoparticles of Fluorescent Conjugated Polymers. Opt. Express 2010, 18, 670-684.

(10) Mauthner, G.; Landfester, K.; Köck, A.; Brückl, H.; Kast, M.; Stepper, C.; List, E. J. W. Inkjet Printed Surface Cell Light-Emitting Devices from a Water-Based Polymer Dispersion. Org. Electron. 2008, 9, 164-170.

(11) Fisslthaler, E.; Sax, S.; Scherf, U.; Mauthner, G.; Moderegger, E.; Landfester, K.; List, E. J. W. Inkjet Printed Polymer Light-Emitting Devices Fabricated by Thermal Embedding of Semiconducting Polymer Nanospheres in an Inert Matrix. Appl. Phys. Lett. 2008, 92, 183305.

(12) Fisslthaler, E.; Blümel, A.; Landfester, K.; Scherf, U.; List, E. J. W. Printing Functional Nanostructures: A Novel Route towards Nanostructuring of Organic Electronic Devices via Soft Embossing, Inkjet Printing and Colloidal Self Assembly of Semiconducting Polymer Nanospheres. Soft Matter 2008, 4, 2448-2453.

(13) Huyal, I. O.; Ozel, T.; Tuncel, D.; Demir, H. V. Quantum Efficiency Enhancement in Film by Making Nanoparticles of Polyfluorene. Opt. Express 2008, 16, 13391-13397.

(14) Park, E.-J.; Erdem, T.; Ibrahimova, V.; Nizamoglu, S.; Demir, H. V.; Tuncel, D. White-emitting Conjugated Polymer Nanoparticles with Cross-linked Shell for Mechanical Stability and Controllable Photometric Properties in Color-Conversion LED Applications. ACS Nano 2011, 5, 2483-2492.

(15) Förster, T. Zwischenmolekulare Energiewanderung und Fluoreszenz. Ann. Phys. 1948, 437, 55-75.

(16) Hayes, G. R.; Samuel, I. D. W.; Phillips, R. T. Exciton Dynamics in Electroluminescent Polymers Studied by Femtosecond TimeResolved Photoluminescence Spectroscopy. Phys. Rev. B 1995, 534, R11569-R11572.

(17) Basham, J. I.; Mor, G. K.; Grimes, C. A. Förster Resonance Energy Transfer in Dye-Sensitized Solar Cells. ACS Nano 2010, 4, $1253-1258$. 
(18) Achermann, M.; Petruska, M. A.; Kos, Š; Smith, D. L.; Koleske, D. L.; Klimov, V. I. Energy-Transfer Pumping of Semiconductor Nanocrystals Using an Epitaxial Quantum-Well. Nature 2004, 429, 642-646.

(19) Basko, D.; La Rocca, G. C.; Bassani, F.; Agranovich, V. M. Förster Energy Transfer from a Semiconductor Quantum Well to an Organic Material Overlayer. Eur. Phys. J. B 1999, 8, 353-362.

(20) Grigalevicius, S.; Forster, M.; Ellinger, S.; Landfester, K.; Scherf, U. Excitation Energy Transfer from Semi-Conducting Polymer Nanoparticles to Surface-Bound Fluorescent Dyes. Macromol. Rapid Commun. 2006, 27, 200-202.

(21) Wu, C.; Peng, H.; Jiang, Y.; McNeill, J. Energy Transfer Mediated Fluorescence from Blended Conjugated Polymer Nanoparticles. J. Phys. Chem. B 2006, 110, 14148-14154.

(22) Huang, C.-Y.; Su, Y.-K.; Chuang, R. W.; Chen, Y.-C.; Huang, T.S.; Wan, C.-T. Tetrachromatic Hybrid White Light-Emitting Diodes and the Energy Transfer Between Conjugated Polymers and CdSe/ ZnS Quantum Dots. J. Electrochem. Soc. 2009, 156, H625-H628.

(23) Belton, C. R.; Itskos, G.; Heliotis, G.; Stavrinou, P. N.; Lagoudakis, P. G.; Lupton, J.; Pereira, S.; Gu, E.; Griffin, C.; Guilhabert, B.; et al. New Light from Hybrid Inorganic-Organic Emitters. J. Phys. D: Appl. Phys. 2008, 41, 094006.

(24) Nguyen, H. M.; Seitz, O.; Aureau, D.; Sra, A.; Nijem, N.; Gartstein, Y. N.; Chabal, Y. J.; Malko, A. V. Spectroscopic Evidence for Nonradiative Energy Transfer between Colloidal CdSe/ZnS Nanocrystals and Functionalized Silicon Substrates. Appl. Phys. Lett. 2011, 98, 161904.

(25) Chanyawadee, S.; Lagoudakis, P. G.; Harley, R. T.; Charlton, M. D. B.; Talapin, D. V.; Lin, S. Increased Color-Conversion Efficiency in Hybrid Light-Emitting Diodes Utilizing Non-Radiative Energy Transfer. Adv. Mater. 2010, 22, 602-606.

(26) Nizamoglu, S.; Guzelturk, B.; Jeon, D.-W.; Lee, I.-H.; Demir, H. V. Efficient Nonradiative Energy Transfer from InGaN/GaN Nanopillars to CdSe/ZnS Core/Shell Nanocrystals. Appl. Phys. Lett. 2011, 98,163108 .

(27) İbrahimova, V.; Ekiz, S.; Gezici, O.; Tuncel, D. Facile Synthesis of Cross-linked Patchy Fluorescent Conjugated Polymer Nanoparticles by Click Reaction. Polym. Chem. 2011, 2, 2818-2824. 